Draft VERSION SEPTEMBER 26, 2019

Typeset using LATEX manuscript style in AASTeX62

\title{
DIFFUSION COEFFICIENT WITH DISPLACEMENT VARIANCE OF ENERGETIC PARTICLES WITH ADIABATIC FOCUSING
}

\author{
J. F. WANG ${ }^{1}$ AND G. QIN ${ }^{1}$ \\ ${ }^{1}$ School of Science, Harbin Institute of Technology, Shenzhen, 518055, China; qingang @ hit.edu.cn
}

\begin{abstract}
The equation $\kappa_{z z}=d \sigma^{2} /(2 d t)$ (hereafter DCDV) is a well-known formula of energetic particles describing the relation of parallel diffusion coefficient $\kappa_{z z}$ with the parallel displacement variance $\sigma^{2}$. In this study, we find that DCDV is only applicable to two kinds of transport equations of isotropic distribution function, one is without cross terms, the other is without convection term. Here, by employing the more general transport equation, i.e., the variable coefficient differential equation derived from the Fokker-Planck equation, a new equation of $\kappa_{z z}$ as a function of $\sigma^{2}$ is obtained. We find that DCDV is the special case of the new equation. In addition, another equation of $\kappa_{z z}$ as a function of $\sigma^{2}$ corresponding to the telegraph equation is also investigated preliminarily.
\end{abstract}

Keywords: diffusion, magnetic fields, scattering, turbulence

\section{INTRODUCTION}

It is very interesting to study energetic charged particle diffusion in the magnetic turbulence superposed on the mean magnetic field in astrophysics (e.g., cosmic ray physics, astrophysical plasmas, and space weather research) and fusion plasma physics (Jokipii 1966; Schlickeiser 2002; Matthaeus et al. 
2003; Shalchi \& Schlickeiser 2005; Shalchi et al. 2006; Qin 2007; Hauff \& Jenko 2008; Shalchi 2009a, 2010; Qin \& Zhang 2014). The interaction process between particles and turbulent magnetic fields is very complicated, and particles and magnetic field lines have the stochastic properties. Therefore, one has to employ methods of statistics to describe the complicated transport of energetic particles (Earl 1974, 1976; Beeck \& Wibberenz 1986; Shalchi 2011; Litvinenko 2012a,b; Shalchi \& Danos 2013; He \& Schlickeiser 2014; Wang et al. 2017a,b; Wang \& Qin 2018) and field line random walk (Matthaeus et al. 1995; Shalchi \& Kourakis 2007; Shalchi \& Qin 2010). Because the background magnetic field $\vec{B}_{0}$ breaks the symmetry of the magnetized plasma, one has to distinguish particle diffusion along and across the largescale magnetic field. One may only consider the parallel diffusion in many cases, when it is much greater than the perpendicular one (see, e.g., Earl 1974, 1976; Beeck \& Wibberenz 1986; Shalchi 2009b, 2011; Litvinenko 2012a,b; Shalchi \& Danos 2013; He \& Schlickeiser 2014; Wang \& Qin 2018).

Various analytical theories of parallel diffusion for energetic charged particles have been developed in the past. The first attempt to resolve the parallel diffusion was the development of quasilinear theory (Jokipii 1966) which corresponds to the first order perturbation theory. Quasilinear theory, however, is problematic and usually does not agree with test-particle simulations (Qin et al. 2002a,b; Shalchi 2009a). With the second order quasilinear theory (SOQLT) developed by Shalchi (2005), by using the Earl's formula (Earl 1974) the parallel diffusion coefficient can be evaluated more accurately (Shalchi et al. 2009a,b; Reimer \& Shalchi 2016). On the other hand, by employing the assumptions and approximations used in NonLinear Guiding Center (NLGC) theory (Matthaeus et al. 2003), Qin (2007) developed an extended parallel diffusion theory.

The mean square displacement $\sigma^{2}=\left\langle(\Delta z)^{2}\right\rangle-\langle(\Delta z)\rangle^{2}$ is defined with the first and second order parallel displacement moments of the isotropic distribution function of the energetic particles. $\sigma^{2}$, describing the spread of possible particle orbits, is one of the fundamental quantities in the parallel transport. In previous research (see, e.g., Shalchi 2009a) the parallel diffusion coefficient $\kappa_{z z}$ is usually described by the following formula (hereafter DCDV)

$$
\kappa_{z z}=\frac{1}{2} \frac{d \sigma^{2}}{d t} .
$$


To study energetic particle diffusion, the background magnetic field $\vec{B}_{0}$, e.g., mean solar wind magnetic field in the interplanetary space, is usually considered as a constant. However, it is clear that the mean solar wind magnetic field is not constant in reality, especially when particles are close to the Sun. It is found that the spatially varying background solar wind magnetic fields lead to the adiabatic focusing effect of charged energetic particle transport and introduce correction to the particle diffusion coefficients (see, e.g., Roelof 1969; Earl 1976; Kunstmann 1979; Beeck \& Wibberenz 1986; Bieber \& Burger 1990; Kóta 2000; Schlickeiser \& Shalchi 2008; Shalchi 2009b, 2011; Litvinenko 2012a,b; Shalchi \& Danos 2013; Wang \& Qin 2016; Wang et al. 2017b; Wang \& Qin 2018). The adiabatic focusing effect causes a convection term in the energetic particles transport equation of the isotropic distribution function, so DCDV might be modified.

In order to address this question, in this paper we derive a new formula for the parallel diffusion coefficient with the parallel displacement variance by modifying DCDV with the adiabatic focusing effect. The paper is organized as follows. In Section 2, from the Fokker-Planck equation with adiabatic focusing effect we derive the isotropic distribution function equation and the telegraph equation. In Section 3 , we evaluate the first and second order moments of the parallel displacement to derive the modified DCDV. In Section 4, some special cases of the modified DCDV are explored. We conclude and summarize our results in Section 5.

\section{EQUATION OF ISOTROPIC DISTRIBUTION FUNCTION}

The standard Fokker-Planck equation, which incorporates the effects of pitch-angle scattering and alongfield adiabatic focusing, is given as (Roelof 1969; Earl 1981)

$$
\frac{\partial f_{0}}{\partial t}+v \mu \frac{\partial f_{0}}{\partial z}=\frac{\partial}{\partial \mu}\left[D_{\mu \mu}(\mu) \frac{\partial f_{0}}{\partial \mu}\right]-\frac{v}{2 L}\left(1-\mu^{2}\right) \frac{\partial f_{0}}{\partial \mu},
$$

which is usually used in previous research (Schlickeiser et al 2007; Shalchi 2011; Litvinenko \& Schlickeiser 2013; Effenberger \& Litvinenko 2014; Malkov \& Sagdeev 2015; Wang \& Qin 2016). Here $f_{0}$ is the isotropic distribution function, $t$ is time, $z$ is the distance along the background magnetic field, $\mu=v_{z} / v$ is the pitch-angle cosine with particle speed $v$ and its z-component $v_{z}, D_{\mu \mu}(\mu)$ is the pitch-angle diffusion coefficient, $L(z)=-B_{0}(z) /\left[d B_{0}(z) / d z\right]$ is the adiabatic focusing characteristic length of the large-scale mag- 
netic field $B_{0}(z)$. In this paper, for simplification we assume that the adiabatic focusing characteristic length is a constant. The source term and the terms related to momentum diffusion and so on are ignored in Equation (2). The more complete form of the Fokker-Planck equation can be found in Schlickeiser (2002).

By introducing the linear phase space density $f(z, \mu, t)=f_{0}(z, \mu, t) / B_{0}$, from Equation (2), the modified Fokker-Planck equation for the distribution function of energetic charged particles can be obtained (Kunstmann 1979; He \& Schlickeiser 2014; Wang et al. 2017b; Wang \& Qin 2018)

$$
\frac{\partial f}{\partial t}+v \mu \frac{\partial f}{\partial z}=\frac{\partial}{\partial \mu}\left[D_{\mu \mu}(\mu) \frac{\partial f}{\partial \mu}-\frac{v}{2 L}\left(1-\mu^{2}\right) f\right] .
$$

Here, $D_{\mu \mu}(\mu)$ is time-independent. However, if the temporal characteristic of the pitch angle diffusion need to be considered, $D_{\mu \mu}=D_{\mu \mu}(\mu, t)$, Equation (3) can be generalized as

$$
\frac{\partial f}{\partial t}+v \mu \frac{\partial f}{\partial z}=\frac{\partial}{\partial \mu}\left[D_{\mu \mu}(\mu, t) \frac{\partial f}{\partial \mu}-\frac{v}{2 L}\left(1-\mu^{2}\right) f\right]
$$

In order for the pitch angle diffusion equation to hold, the characteristic time of the pitch angle diffusion has to be much less than the characteristic time of $D_{\mu \mu}(\mu, t)$. Equation (4) is the starting point of the research in this paper.

With strong pitch-angle scattering, the gyro-tropic cosmic-ray phase space density $f(\vec{x}, \mu, t)$ can be split into the dominant isotropic part $F(\vec{x}, t)$ and the subordinate anisotropic part $g(\vec{x}, \mu, t)$ (see, e.g., Schlickeiser et al 2007; Schlickeiser \& Shalchi 2008; He \& Schlickeiser 2014; Wang et al. 2017b; Wang \& Qin 2018)

$$
f(\vec{x}, \mu, t)=F(\vec{x}, t)+g(\vec{x}, \mu, t)
$$

with

$$
F(\vec{x}, t)=\frac{1}{2} \int_{-1}^{1} d \mu f(\vec{x}, \mu, t)
$$

and

$$
\int_{-1}^{1} d \mu g(\vec{x}, \mu, t)=0
$$

2.1. The variable coefficient differential equation of the isotropic distribution function $F(z, t)$ 
In this subsection, by employing the method in Wang \& Qin (2018) we derive the formula of the variable coefficient differential equation of the isotropic distribution function $F(z, t)$, and the derivation are similar to Equation (22) in Wang \& Qin (2018).

Integration of Equation (4) over $\mu$ gives

$$
\frac{\partial F}{\partial t}+\frac{v}{2} \frac{\partial}{\partial z} \int_{-1}^{1} \mu g d \mu=0
$$

Next, integrating Equation (4) over $\mu$ from -1 to $\mu$, and using the regularity $D_{\mu \mu}(\mu= \pm 1)=0$ we can find

$$
\frac{\partial F}{\partial t}(\mu+1)+\frac{\partial}{\partial t} \int_{-1}^{\mu} d v g+\frac{v\left(\mu^{2}-1\right)}{2} \frac{\partial F}{\partial z}+v \frac{\partial}{\partial z} \int_{-1}^{\mu} d v v g=D_{\mu \mu}(\mu, t) \frac{\partial g}{\partial \mu}-\frac{v\left(1-\mu^{2}\right)}{2 L} F-\frac{v\left(1-\mu^{2}\right)}{2 L} g
$$

By subtracting Equation (8) from (9) we can find the following equation

$$
\begin{gathered}
\frac{\partial F}{\partial t} \mu+\frac{\partial}{\partial t} \int_{-1}^{\mu} d v g+\frac{v\left(\mu^{2}-1\right)}{2} \frac{\partial F}{\partial z}+v \frac{\partial}{\partial z} \int_{-1}^{\mu} d v v g-\frac{v}{2} \frac{\partial}{\partial z} \int_{-1}^{1} \mu g d \mu \\
=D_{\mu \mu}(\mu, t) \frac{\partial g}{\partial \mu}-\frac{v\left(1-\mu^{2}\right)}{2 L} F-\frac{v\left(1-\mu^{2}\right)}{2 L} g
\end{gathered}
$$

After a straightforward algebra, Equation (10) reduces to the following form

$$
\frac{\partial g}{\partial \mu}-\frac{v\left(1-\mu^{2}\right) g}{2 L D_{\mu \mu}(\mu, t)}+\frac{v\left(1-\mu^{2}\right)}{2 D_{\mu \mu}(\mu, t)}\left(\frac{\partial F}{\partial z}-\frac{F}{L}\right)=\Phi(\mu, t)
$$

with

$$
\Phi(\mu, t)=\frac{1}{D_{\mu \mu}(\mu, t)}\left[\left(\frac{\partial F}{\partial t} \mu+\frac{\partial}{\partial t} \int_{-1}^{\mu} g d v\right)+\frac{v}{2} \frac{\partial}{\partial z}\left(2 \int_{-1}^{\mu} d v v g-\int_{-1}^{1} d \mu \mu g\right)\right] .
$$

Equation (11) can be rewritten as

$$
\frac{\partial}{\partial \mu}\left\{\left[g(\mu, t)-L\left(\frac{\partial F}{\partial z}-\frac{F}{L}\right)\right] e^{-M(\mu, t)}\right\}=e^{-M(\mu, t)} \Phi(\mu, t)
$$

with

$$
M(\mu, t)=\frac{v}{2 L} \int_{-1}^{\mu} d v \frac{1-v^{2}}{D_{v v}(v, t)}
$$

In additon, with the same procedure used in Wang \& Qin (2018), the anisotropic distribution function can be obtained as following

$$
g(\mu, t)=L\left(\frac{\partial F}{\partial z}-\frac{F}{L}\right)\left[1-\frac{2 e^{M(\mu, t)}}{\int_{-1}^{1} d \mu e^{M(\mu, t)}}\right]+e^{M(\mu, t)}\left[R(\mu, t)-\frac{\int_{-1}^{1} d \mu e^{M(\mu, t)} R(\mu, t)}{\int_{-1}^{1} d \mu e^{M(\mu, t)}}\right]
$$


with

$$
R(\mu, t)=\int_{-1}^{\mu} d v e^{-M(v, t)} \Phi(v, t)
$$

Equations (15) and (16) contain the effect from the term on the right hand side of Equation (13). Combining Equations (12), (15) and (16) gives the iterative function of $g(\mu, t)$,

$$
\begin{aligned}
g(\mu, t)= & L\left(\frac{\partial F}{\partial z}-\frac{F}{L}\right)\left[1-\frac{2 e^{M(\mu, t)}}{\int_{-1}^{1} d \mu e^{M(\mu, t)}}\right]+e^{M(\mu, t)}\left\{\int _ { - 1 } ^ { \mu } d v e ^ { - M ( v , t ) } \frac { 1 } { D _ { v v } ( v , t ) } \left[\left(\frac{\partial F}{\partial t} v+\frac{\partial}{\partial t} \int_{-1}^{v} g(\rho, t) d \rho\right)\right.\right. \\
& \left.+\frac{v}{2} \frac{\partial}{\partial z}\left(2 \int_{-1}^{v} d \rho \rho g(\rho, t)-\int_{-1}^{1} d \mu \mu g(\mu, t)\right)\right] \\
& -\frac{1}{\int_{-1}^{1} d \mu e^{M(\mu, t)}} \int_{-1}^{1} d \mu e^{M(\mu, t)} \int_{-1}^{\mu} d v e^{-M(v, t)} \frac{1}{D_{v v}(v, t)}\left[\left(\frac{\partial F}{\partial t} v+\frac{\partial}{\partial t} \int_{-1}^{v} g(\rho, t) d \rho\right)\right. \\
& \left.\left.+\frac{v}{2} \frac{\partial}{\partial z}\left(2 \int_{-1}^{\mu} d v v g(v, t)-\int_{-1}^{1} d \mu \mu g(\mu, t)\right)\right]\right\} .
\end{aligned}
$$

By iterating Equation (17), we obtain the formula of $g(\mu, t)$ as

$$
g(\mu, t)=\sum_{m, n} \epsilon_{m, n}(t) \frac{\partial^{m+n}}{\partial t^{m} \partial z^{n}} F
$$

with the time dependent coefficients $\epsilon_{m, n}(t)$. Here $m, n=0,1,2,3, \cdots$. Furthermore, considering Equation (12), (15) and Equation (16), we can write $R(\mu, t)$ as

$$
R(\mu, t)=\sum_{m, n} \chi_{m, n}(t) \frac{\partial^{m+n}}{\partial t^{m} \partial z^{n}} F
$$

with the time dependent coefficients $\chi_{m, n}(t)$ and $m, n=0,1,2,3, \cdots$. The coefficients $\chi_{m, n}(t)$ in the latter equation is related to the coefficients $\epsilon_{m, n}(t)$ in Equation (18).

In order to obtain the differential equation of isotropic distribution function, we need to get the following formula by using Equation (8)

$$
\int_{-1}^{1} d \mu \mu g(\mu, t)=-2 \frac{\int_{-1}^{1} d \mu \mu e^{M(\mu, t)}}{\int_{-1}^{1} d \mu e^{M(\mu, t)}}\left(\frac{\partial F}{\partial z}-\frac{F}{L}\right) L+\int_{-1}^{1} d \mu \mu e^{M(\mu, t)}\left[R(\mu, t)-\frac{\int_{-1}^{1} d \mu e^{M(\mu, t)} R(\mu, t)}{\int_{-1}^{1} d \mu e^{M(\mu, t)}}\right] .
$$

By inserting Equation (20) into Equation (8) one can obtain

$$
\frac{\partial F}{\partial t}-\frac{\partial}{\partial z}\left[\nu L \frac{\int_{-1}^{1} d \mu \mu e^{M(\mu, t)}}{\int_{-1}^{1} d \mu e^{M(\mu, t)}}\left(\frac{\partial F}{\partial z}-\frac{F}{L}\right)\right]+\Lambda(z, t)=0
$$


with

$$
\Lambda(z, t)=\frac{v}{2} \int_{-1}^{1} d \mu \mu e^{M(\mu, t)}\left[\frac{\partial R(\mu, t)}{\partial z}-\frac{\int_{-1}^{1} d \mu \frac{\partial R(\mu, t)}{\partial z} e^{M(\mu, t)}}{\int_{-1}^{1} d \mu e^{M(\mu, t)}}\right] .
$$

By inserting Equation (19) into Equation (22) we can find

$$
\Lambda(z, t)=\sum_{m, n} \eta_{m, n}(t) \frac{\partial^{m+n}}{\partial t^{m} \partial z^{n}} F
$$

with the coefficients $\eta_{m, n}(t)$ and $m, n=0,1,2,3, \cdots$. Thus, Equation (21) can be written as

$$
\frac{\partial F}{\partial t}-\frac{\partial}{\partial z}\left[v L \frac{\int_{-1}^{1} d \mu \mu e^{M(\mu, t)}}{\int_{-1}^{1} d \mu e^{M(\mu, t)}}\left(\frac{\partial F}{\partial z}-\frac{F}{L}\right)\right]+\sum_{m, n} \eta_{m, n}(t) \frac{\partial^{m+n}}{\partial t^{m} \partial z^{n}} F=0
$$

Now we get a variable coefficient linear differential equation of the isotropic distribution function.

\subsection{The specific form of the variable coefficient differential equation}

By employing the method in Wang \& Qin (2018), one can rewrite Equation (24) as

$$
\begin{aligned}
\frac{\partial F}{\partial t}= & -\kappa_{z}(t) \frac{\partial F}{\partial z}+\kappa_{z z}(t) \frac{\partial^{2} F}{\partial z^{2}}+\left(\kappa_{z z z}(t) \frac{\partial^{3} F}{\partial z^{3}}+\kappa_{z z z z}(t) \frac{\partial^{4} F}{\partial z^{4}}+\cdots\right)+\left(\kappa_{t z}(t) \frac{\partial^{2} F}{\partial t \partial z}+\kappa_{t t z}(t) \frac{\partial^{3} F}{\partial t^{2} \partial z}+\kappa_{t t t z}(t) \frac{\partial^{4} F}{\partial t^{3} \partial z}+\cdots\right) \\
& +\left(\kappa_{t z z}(t) \frac{\partial^{3} F}{\partial t \partial z^{2}}+\kappa_{t t z z}(t) \frac{\partial^{4} F}{\partial t^{2} \partial z^{2}}+\kappa_{t t t z z}(t) \frac{\partial^{5} F}{\partial t^{3} \partial z^{2}}+\cdots\right)+\cdots \cdots,
\end{aligned}
$$

here, $\kappa_{z}(t), \kappa_{z z}(t), \kappa_{z z z}(t), \kappa_{z z z z}(t), \cdots \cdots, \kappa_{t z}(t), \kappa_{t t z}(t), \kappa_{t t t z}(t), \cdots \cdots, \kappa_{t z z}(t), \kappa_{t t z z}(t), \kappa_{t t t z z}(t), \cdots \cdots$ are all time dependent. This equation determines the transport regimes by the specific forms of its coefficient. For example, as shown in Wang \& Qin (2018), $\kappa_{z}(t)$ is the coefficient of the convection term

$$
\kappa_{z}(t)=v \frac{\int_{-1}^{1} d \mu \mu e^{M(\mu, t)}}{\int_{-1}^{1} d \mu e^{M(\mu, t)}}
$$

and $\kappa_{z z}(t)$ is the coefficient of the parallel diffusion term

$$
\begin{aligned}
\kappa_{z z}(t)= & \nu L \frac{\int_{-1}^{1} d \mu \mu e^{M(\mu, t)}}{\int_{-1}^{1} d \mu e^{M(\mu, t)}}+\frac{v^{2}}{2}\left\{\int_{-1}^{1} d \mu \mu e^{M(\mu, t)} \int_{-1}^{\mu} d \nu \frac{e^{-M(v, t)}}{D_{v v}(v, t)}\left[\int_{-1}^{v} d \rho \rho\left(1-\frac{2 e^{M(\rho, t)}}{\int_{-1}^{1} d \mu e^{M(\mu, t)}}\right)+\int_{-1}^{1} d \mu \mu \frac{e^{M(\mu, t)}}{\int_{-1}^{1} d \mu e^{M(\mu, t)}}\right]\right. \\
& \left.-\frac{\int_{-1}^{1} d \mu \mu e^{M(\mu, t)}}{\int_{-1}^{1} d \mu e^{M(\mu, t)}} \int_{-1}^{1} d \mu e^{M(\mu, t)} \int_{-1}^{\mu} d v \frac{e^{-M(v, t)}}{D_{v v}(v, t)}\left[\int_{-1}^{v} d \rho \rho\left(1-\frac{2 e^{M(\rho, t)}}{\int_{-1}^{1} d \mu e^{M(\mu, t)}}\right)+\int_{-1}^{1} d \mu \mu \frac{e^{M(\mu, t)}}{\int_{-1}^{1} d \mu e^{M(\mu, t)}}\right]\right\} . \quad(27)
\end{aligned}
$$

We can similarly obtain other time-dependent coefficients in Equation (25). 


\subsection{Telegraph equation of the isotropic distribution function $F(z, t)$}

The diffusion convection equation is frequently used in previous research (see, e.g., Shalchi 2009a). But the accuracy of the diffusion approximation is limited because the signal speed is infinite in the diffusion limit. Recently, an improved description of the energetic particle transport is provided by the telegraph equation, characterized by a finite signal propagation speed (Kóta 1994; Pauls \& Burger 1994; Schwadron \& Gombosi 2010; Porrà \& Masoliver 1997; Zank et al. 2000; Fedorov \& Shakhov 2003; Kaghashvili et al. 2004; Litvinenko \& Schlickeiser 2013; Effenberger \& Litvinenko 2014; Litvinenko et al. 2015; Litvinenko \& Noble 2016). Using the iterative method in Litvinenko \& Schlickeiser (2013), one can obtain the telegraph equation from Equation (25)

$$
\frac{\partial F}{\partial t}+\tau(t) \frac{\partial^{2} F}{\partial t^{2}}+\kappa_{z}(t) \frac{\partial F}{\partial z}=\kappa_{z z}(t) \frac{\partial^{2} F}{\partial z^{2}}
$$

with

$$
\tau(t)=-\frac{\kappa_{t z z}(t)}{\kappa_{z z}(t)}
$$

Equation (25) can be rewritten as

$$
\begin{aligned}
\frac{\partial F}{\partial z}= & \frac{1}{\kappa_{z}(t)}\left[-\frac{\partial F}{\partial t}+\kappa_{z z}(t) \frac{\partial^{2} F}{\partial z^{2}}+\left(\kappa_{z z z}(t) \frac{\partial^{3} F}{\partial z^{3}}+\kappa_{z z z z}(t) \frac{\partial^{4} F}{\partial z^{4}}+\cdots\right)+\left(\kappa_{t z}(t) \frac{\partial^{2} F}{\partial t \partial z}+\kappa_{t t z}(t) \frac{\partial^{3} F}{\partial t^{2} \partial z}+\kappa_{t t t z}(t) \frac{\partial^{4} F}{\partial t^{3} \partial z}+\cdots\right)\right. \\
& \left.+\left(\kappa_{t z z}(t) \frac{\partial^{3} F}{\partial t \partial z^{2}}+\kappa_{t t z z}(t) \frac{\partial^{4} F}{\partial t^{2} \partial z^{2}}+\kappa_{t t t z z}(t) \frac{\partial^{5} F}{\partial t^{3} \partial z^{2}}+\cdots\right)+\cdots \cdots\right] .
\end{aligned}
$$

Inserting Equation (30) into the convection term $\kappa_{t z} \partial^{2} F / \partial t \partial z$ in Equation (25) and neglecting the higher order derivative terms, we can obtain another version of the telegraph equation,

$$
\frac{\partial F}{\partial t}+\tau^{\prime}(t) \frac{\partial^{2} F}{\partial t^{2}}+\kappa_{z}(t) \frac{\partial F}{\partial z}=\kappa_{z z}(t) \frac{\partial^{2} F}{\partial z^{2}}
$$

with

$$
\tau^{\prime}(t)=\frac{\kappa_{t z}(t)}{\kappa_{z}(t)}
$$

We find that the only difference between Equations (31) and (28) is caused by the fact that $\tau(t)$ is not equal to $\tau^{\prime}(t)$. 


\section{THE MODIFIED FORMULA OF DIFFUSION COEFFICIENT WITH DISPLACEMENT VARIANC}

In the following, we derive the modified formula of the parallel diffusion coefficient with the parallel displacement variance induced by the adiabatic focusing (hereafter MDCDV).

\subsection{MDCDV for the variable coefficient differential equation}

By multiplying Equation (25) with $\langle(\Delta z)\rangle$ and integrating the result, one can find

$$
\begin{aligned}
\frac{d}{d t}\langle(\Delta z)\rangle= & \int_{-\infty}^{\infty} d z(\Delta z) \frac{\partial F}{\partial t} \\
= & \int_{-\infty}^{\infty} d z(\Delta z)\left[\left(-\kappa_{z}(t) \frac{\partial F}{\partial z}+\kappa_{z z}(t) \frac{\partial^{2} F}{\partial z^{2}}+\kappa_{z z z}(t) \frac{\partial^{3} F}{\partial z^{3}}+\kappa_{z z z z}(t) \frac{\partial^{4} F}{\partial z^{4}}+\cdots+\kappa_{z \cdots z}(t) \frac{\partial^{n} F}{\partial z^{n}}+\cdots\right)\right. \\
& +\left(\kappa_{t z}(t) \frac{\partial^{2} F}{\partial t \partial z}+\kappa_{t t z}(t) \frac{\partial^{3} F}{\partial t^{2} \partial z}+\kappa_{t t t z}(t) \frac{\partial^{4} F}{\partial t^{3} \partial z}+\cdots+\kappa_{t \cdots t z}(t) \frac{\partial^{n+1} F}{\partial t^{n} \partial z}+\cdots\right) \\
& \left.+\left(\kappa_{t z z}(t) \frac{\partial^{3} F}{\partial t \partial z^{2}}+\kappa_{t t z z}(t) \frac{\partial^{4} F}{\partial t^{2} \partial z^{2}}+\cdots+\kappa_{t t z z}(t) \frac{\partial^{4} F}{\partial t^{2} \partial z^{2}}+\kappa_{t \cdots t z z}(t) \frac{\partial^{n+2} F}{\partial t^{n} \partial z^{2}}+\cdots\right)+\cdots \cdots\right]
\end{aligned}
$$

To proceed, we have to obtain the integrals on the right hand side of the latter equation with the following conditions

$$
\begin{aligned}
& F(z= \pm \infty)=0, \\
& \frac{\partial^{n} F}{\partial z^{n}}(z= \pm \infty)=0 \quad n=1,2,3, \cdots .
\end{aligned}
$$

By performing integration in parts one can obtain

$$
\begin{aligned}
& \int_{-\infty}^{\infty} d z(\Delta z)\left(-\kappa_{z}(t) \frac{\partial F}{\partial z}\right)=-\kappa_{z}(t) \int_{-\infty}^{\infty} d z(\Delta z) \frac{\partial F}{\partial z}=\kappa_{z}(t), \\
& \int_{-\infty}^{\infty} d z(\Delta z)\left(\kappa_{z z}(t) \frac{\partial^{2} F}{\partial z^{2}}\right)=\kappa_{z z}(t) \int_{-\infty}^{\infty} d z(\Delta z) \frac{\partial^{2} F}{\partial z^{2}}=0 .
\end{aligned}
$$

We can also find that the integrals are equal to zero if the order of spatial derivative of the integrands is higher than one,

$$
\int_{-\infty}^{\infty} d z(\Delta z)\left(\kappa_{z \cdots z}(t) \frac{\partial^{n} F}{\partial z^{n}}\right)=\kappa_{z \cdots z}(t) \int_{-\infty}^{\infty} d z(\Delta z) \frac{\partial^{n} F}{\partial z^{n}}=0 \quad n=2,3, \cdots
$$

It is also easy to find the following result by integration by parts

$$
\int_{-\infty}^{\infty} d z(\Delta z)\left(\kappa_{z \cdots z}(t) \frac{\partial^{n+m} F}{\partial t^{m} \partial z^{n}}\right)=\kappa_{z \cdots z}(t) \frac{d^{m}}{d t^{m}} \int_{-\infty}^{\infty} d z(\Delta z) \frac{\partial^{n} F}{\partial z^{n}}=0 \quad n=1,2,3, \cdots, \quad m=1,2,3, \cdots
$$


Inserting Equations (36) - (39) into Equation (33), one can obtain the following formula

$$
\frac{d}{d t}\langle(\Delta z)\rangle=\kappa_{z}(t)
$$

Furthermore, analogous to Equation (33), the formula of second order moment of the parallel displacement can be written as

$$
\begin{aligned}
\frac{d}{d t}\left\langle(\Delta z)^{2}\right\rangle= & \int_{-\infty}^{\infty} d z(\Delta z)^{2} \frac{\partial F}{\partial t} \\
= & \int_{-\infty}^{\infty} d z(\Delta z)^{2}\left[\left(-\kappa_{z}(t) \frac{\partial F}{\partial z}+\kappa_{z z}(t) \frac{\partial^{2} F}{\partial z^{2}}+\kappa_{z z z}(t) \frac{\partial^{3} F}{\partial z^{3}}+\kappa_{z z z z}(t) \frac{\partial^{4} F}{\partial z^{4}}+\cdots+\kappa_{z \cdots z}(t) \frac{\partial^{n} F}{\partial z^{n}}+\cdots\right)\right. \\
& +\left(\kappa_{t z} \frac{\partial^{2} F}{\partial t \partial z}+\kappa_{t t z}(t) \frac{\partial^{3} F}{\partial t^{2} \partial z}+\kappa_{t t t z}(t) \frac{\partial^{4} F}{\partial t^{3} \partial z}+\cdots+\kappa_{t \cdots t z}(t) \frac{\partial^{n+1} F}{\partial t^{n} \partial z}+\cdots\right) \\
& \left.+\left(\kappa_{t z z}(t) \frac{\partial^{3} F}{\partial t \partial z^{2}}+\kappa_{t t z z}(t) \frac{\partial^{4} F}{\partial t^{2} \partial z^{2}}+\kappa_{t t t z z}(t) \frac{\partial^{5} F}{\partial t^{3} \partial z^{2}}+\cdots+\kappa_{t \cdots t z z}(t) \frac{\partial^{n+2} F}{\partial t^{n} \partial z^{2}}+\cdots\right)+\cdots \cdots\right]
\end{aligned}
$$

The integrals on the right hand side of the latter equation can be easily found by part integral

$$
\begin{aligned}
& \int_{-\infty}^{\infty} d z(\Delta z)^{2}\left(-\kappa_{z}(t) \frac{\partial F}{\partial z}\right)=-\kappa_{z}(t) \int_{-\infty}^{\infty} d z(\Delta z)^{2} \frac{\partial F}{\partial z}=2 \kappa_{z}(t)\langle(\Delta z)\rangle \\
& \int_{-\infty}^{\infty} d z(\Delta z)^{2}\left(\kappa_{z z}(t) \frac{\partial^{2} F}{\partial z^{2}}\right)=\kappa_{z z}(t) \int_{-\infty}^{\infty} d z(\Delta z)^{2} \frac{\partial^{2} F}{\partial z^{2}}=2 \kappa_{z z}(t) \\
& \int_{-\infty}^{\infty} d z(\Delta z)^{2}\left(\kappa_{z \cdots z}(t) \frac{\partial^{n} F}{\partial z^{n}}\right)=\kappa_{z \cdots z}(t) \int_{-\infty}^{\infty} d z(\Delta z)^{2} \frac{\partial^{n} F}{\partial z^{n}}=0 \quad n=3,4,5, \cdots \\
& \int_{-\infty}^{\infty} d z(\Delta z)^{2}\left(\kappa_{t \cdots t z}(t) \frac{\partial^{n+1} F}{\partial t^{n} \partial z}\right)=\kappa_{t \cdots+t z}(t) \frac{d^{n}}{d t^{n}} \int_{-\infty}^{\infty} d z(\Delta z)^{2} \frac{\partial F}{\partial z}=-2 \kappa_{t \cdots t z}(t) \frac{d^{n}}{d t^{n}}\langle(\Delta z)\rangle \quad n=1,2,3, \cdots \\
& \int_{-\infty}^{\infty} d z(\Delta z)^{2}\left(\kappa_{t \cdots t z \cdots z}(t) \frac{\partial^{n+m} F}{\partial t^{n} \partial z^{m}}\right)=\kappa_{t \cdots t z \cdots z}(t) \frac{d^{n}}{d t^{n}} \int_{-\infty}^{\infty} d z(\Delta z)^{2} \frac{\partial F^{m}}{\partial z^{m}}=0 \quad n=1,2,3, \cdots, m=2,3, \cdots,
\end{aligned}
$$

With Equations (42)-(46), Equation (41) becomes

$$
\frac{d}{d t}\left\langle(\Delta z)^{2}\right\rangle=2 \kappa_{z z}(t)+2\left[\kappa_{z}(t)\langle(\Delta z)\rangle-\kappa_{t z}(t) \frac{d}{d t}\langle(\Delta z)\rangle-\kappa_{t z}(t) \frac{d^{2}}{d t^{2}}\langle(\Delta z)\rangle-\kappa_{t t t z}(t) \frac{d^{3}}{d t^{3}}\langle(\Delta z)\rangle-\cdots \cdots\right] .
$$

The latter equation can be rewritten as

$$
\kappa_{z z}(t)=\frac{1}{2} \frac{d}{d t}\left\langle(\Delta z)^{2}\right\rangle+C(t)
$$

with

$$
C(t)=-\kappa_{z}(t)\langle(\Delta z)\rangle+\kappa_{t z}(t) \frac{d}{d t}\langle(\Delta z)\rangle+\kappa_{t t z}(t) \frac{d^{2}}{d t^{2}}\langle(\Delta z)\rangle+\kappa_{t t t z}(t) \frac{d^{3}}{d t^{3}}\langle(\Delta z)\rangle+\cdots \cdots .
$$


Here, $C(t)$ is related to convection of energetic particles.

By employing formula $\sigma^{2}=\left\langle(\Delta z)^{2}\right\rangle-\langle(\Delta z)\rangle^{2}$ and Equation (40) we can obtain

$$
\kappa_{z z}(t)=\frac{1}{2} \frac{d \sigma^{2}}{d t}+\left[\kappa_{t z}(t) \frac{d}{d t}\langle(\Delta z)\rangle+\kappa_{t t z}(t) \frac{d^{2}}{d t^{2}}\langle(\Delta z)\rangle+\kappa_{t t t z}(t) \frac{d^{3}}{d t^{3}}\langle(\Delta z)\rangle+\cdots \cdots\right] .
$$

Inserting Equation (40) into the latter equation one can find

$$
\kappa_{z z}(t)=\frac{1}{2} \frac{d \sigma^{2}}{d t}+\left[\kappa_{t z}(t) \kappa_{z}(t)+\kappa_{t t z}(t) \frac{d}{d t} \kappa_{z}(t)+\kappa_{t t t z}(t) \frac{d^{2}}{d t^{2}} \kappa_{z}(t)+\cdots \cdots\right] .
$$

The latter equation can be rewritten as

$$
\frac{d \sigma^{2}}{d t}=2 \kappa_{z z}(t)-\left[2 \kappa_{t z}(t) \kappa_{z}(t)+2 \kappa_{t t z}(t) \frac{d}{d t} \kappa_{z}(t)+2 \kappa_{t t t z}(t) \frac{d^{2}}{d t^{2}} \kappa_{z}(t)+\cdots \cdots\right] .
$$

Equation (50) or (52) is the modified DCDV (MDCDV). In comparison with the well-known Equation (1) (represented by DCDV), one can find that MDCDV is a more general formula. That is, DCDV is the special case of MDCDV when the cross terms with the first order spatial derivative or the convection term are equal to zero.

Because the coefficients $\kappa_{z}(t), \kappa_{z z}(t), \kappa_{t z}(t), \cdots \cdots$ are all time dependent, the relation $\sigma^{2} \propto t$ does not hold for MDCDV. Therefore, the transport described by MDCDV is not in diffusion regime. Since Equation (52) is derived from Equation (25), the transport described by Equation (25) is also not in diffusion regime.

\subsection{MDCDV for the telegraph equation}

For the telegraph equation derived in Litvinenko \& Schlickeiser (2013) the corresponding first and second order moments of the parallel displacement can be written as, respectively

$$
\begin{aligned}
& \frac{d}{d t}\langle(\Delta z)\rangle=\kappa_{z}(t)-\tau(t) \frac{d^{2}}{d t^{2}}\langle(\Delta z)\rangle, \\
& \frac{d}{d t}\left\langle(\Delta z)^{2}\right\rangle=2 \kappa_{z}(t)\langle(\Delta z)\rangle+2 \kappa_{z z}(t)-\tau(t) \frac{d^{2}}{d t^{2}}\left\langle(\Delta z)^{2}\right\rangle .
\end{aligned}
$$

By combining the latter equations one can derive with $\sigma^{2}=\left\langle(\Delta z)^{2}\right\rangle-\langle(\Delta z)\rangle^{2}$

$$
\kappa_{z z}(t)=\frac{1}{2} \frac{d \sigma^{2}}{d t}+\tau(t)\left[\frac{1}{2} \frac{d^{2}}{d t^{2}}\left\langle(\Delta z)^{2}\right\rangle-\langle(\Delta z)\rangle \frac{d^{2}}{d t^{2}}\langle(\Delta z)\rangle\right] .
$$

Inserting Equation (32) into the latter equation, we can obtain

$$
\kappa_{z z}(t)=\frac{1}{2} \frac{d \sigma^{2}}{d t}-\frac{\kappa_{t z z}(t)}{\kappa_{z z}(t)}\left[\frac{d^{2}}{d t^{2}}\left\langle(\Delta z)^{2}\right\rangle-\langle(\Delta z)\rangle \frac{d^{2}}{d t^{2}}\langle(\Delta z)\rangle\right] .
$$


By subtracting Equation (56) from Equation (51) we can obtain the error formula

$$
E(t)=\left[\kappa_{t z}(t) \kappa_{z}(t)+\kappa_{t t z}(t) \frac{d}{d t} \kappa_{z}(t)+\kappa_{t t t z}(t) \frac{d^{2}}{d t^{2}} \kappa_{z}(t)+\cdots \cdots\right]+\frac{\kappa_{t z z}(t)}{\kappa_{z z}(t)}\left[\frac{d^{2}}{d t^{2}}\left\langle(\Delta z)^{2}\right\rangle-\langle(\Delta z)\rangle \frac{d^{2}}{d t^{2}}\langle(\Delta z)\rangle\right],
$$

which comes from the iteration operation used in the derivation of telegraph equation. In the future, this formula will be explored in detail.

In addition, Equation (53) can be rewritten as

$$
\tau(t) \frac{d^{2}}{d t^{2}}\langle(\Delta z)\rangle+\frac{d}{d t}\langle(\Delta z)\rangle=\kappa_{z}(t)
$$

If the coefficients $\tau(t)$ and $\kappa_{z}(t)$ can be determined, the latter equation might be solved. Similarily, Equation (54) can be rewritten as

$$
\tau(t) \frac{d^{2}}{d t^{2}}\left\langle(\Delta z)^{2}\right\rangle+\frac{d}{d t}\left\langle(\Delta z)^{2}\right\rangle=2 \kappa_{z}(t)\langle(\Delta z)\rangle+2 \kappa_{z z}(t)
$$

Combining the solution of Equation (58) and the coefficients $\tau(t)$ and $\kappa_{z}(t)$ with the latter equation, the formula of $\left\langle(\Delta z)^{2}\right\rangle$ might be got. Some solutions of Equations (58) and (59) will be investigated in the future.

\subsection{MDCDV for telegraph Equation (31)}

Similarily, for telegraph Equation (31) the first and second moments can be got as follows

$$
\begin{aligned}
& \frac{d}{d t}\langle(\Delta z)\rangle=\kappa_{z}(t)-\tau^{\prime}(t) \frac{d^{2}}{d t^{2}}\langle(\Delta z)\rangle, \\
& \frac{d}{d t}\left\langle(\Delta z)^{2}\right\rangle=2 \kappa_{z}(t)\langle(\Delta z)\rangle+2 \kappa_{z z}(t)-\tau^{\prime}(t) \frac{d^{2}}{d t^{2}}\left\langle(\Delta z)^{2}\right\rangle .
\end{aligned}
$$

The corresponding MDCDV becomes

$$
\kappa_{z z}(t)=\frac{1}{2} \frac{d \sigma^{2}}{d t}+\frac{\kappa_{t z}(t)}{\kappa_{z}(t)}\left[\frac{d^{2}}{d t^{2}}\left\langle(\Delta z)^{2}\right\rangle-\langle(\Delta z)\rangle \frac{d^{2}}{d t^{2}}\langle(\Delta z)\rangle\right] .
$$

\section{SPECIAL CASES}

In the following, we investigate the special cases of MDCDV (see Equation (50)), which correspond to the simplified versions of Equation (25). 


\subsection{Case 1: constant coefficient differential equation}

If the temporal effect of the pitch-angle diffusion coefficient $D_{\mu \mu}$ can be ignored, i.e., $D_{\mu \mu}=D_{\mu \mu}(\mu)$, all the coefficients in Equation (25) are constant. Therefore, Equation (25) is simplified as

$$
\begin{aligned}
\frac{\partial F}{\partial t}= & \left(-\kappa_{z} \frac{\partial F}{\partial z}+\kappa_{z z} \frac{\partial^{2} F}{\partial z^{2}}+\kappa_{z z z} \frac{\partial^{3} F}{\partial z^{3}}+\kappa_{z z z z} \frac{\partial^{4} F}{\partial z^{4}}+\cdots\right)+\left(\kappa_{t z} \frac{\partial^{2} F}{\partial t \partial z}+\kappa_{t t z} \frac{\partial^{3} F}{\partial t^{2} \partial z}+\kappa_{t t t z} \frac{\partial^{4} F}{\partial t^{3} \partial z}+\cdots\right) \\
& +\left(\kappa_{t z z} \frac{\partial^{3} F}{\partial t \partial z^{2}}+\kappa_{t t z z} \frac{\partial^{4} F}{\partial t^{2} \partial z^{2}}+\kappa_{t t t z z} \frac{\partial^{5} F}{\partial t^{3} \partial z^{2}}+\cdots\right)+\cdots \cdots
\end{aligned}
$$

This is a constant coefficient linear differential equation of the isotropic distribution function. The first and second order moments of the parallel displacement can be found as

$$
\begin{aligned}
& \frac{d}{d t}\langle(\Delta z)\rangle=\kappa_{z}=\text { constant }, \\
& \frac{d}{d t}\left\langle(\Delta z)^{2}\right\rangle=2 \kappa_{z}\langle(\Delta z)\rangle+2 \kappa_{z z}-2 \kappa_{t z} \kappa_{z} .
\end{aligned}
$$

By combining the latter two formulas one can derive

$$
\kappa_{z z}=\frac{1}{2} \frac{d \sigma^{2}}{d t}+\kappa_{t z} \kappa_{z}
$$

We can find that DCDV is the special case of the latter equation when $\kappa_{t z} \kappa_{z}=0$.

Because the coefficients $\kappa_{z}, \kappa_{z z}$ and $\kappa_{t z}$ are all constants, Equation (66) can be rewritten as

$$
\frac{d \sigma^{2}}{d t}=2 \kappa_{z z}-2 \kappa_{t z} \kappa_{z}=\text { constant }
$$

By setting the initial value of $\sigma^{2}$ as zero, from the latter equation we can obtain

$$
\sigma^{2}=\alpha t
$$

with

$$
\alpha=2 \kappa_{t z} \kappa_{z}+2 \kappa_{z z}=\text { constant }
$$

Equation (68) describes diffusive regime. Therefore, for $D_{\mu \mu}=D_{\mu \mu}(\mu)$ the constant coefficient linear differential equation of the isotropic distribution function (see Equation (63)) can describe the diffusion of the energetic particles. If $\kappa_{z}$ or $\kappa_{t z}$ can be ignored, DCDV (Equation (1)) is obtained. 


\subsection{Case 2: diffusion convection equation}

If Equation (63) is simplified to the diffusion convection equation

$$
\frac{\partial F}{\partial t}=-\kappa_{z} \frac{\partial F}{\partial z}+\kappa_{z z} \frac{\partial^{2} F}{\partial z^{2}}
$$

Equation (66) becomes DCDV.

\subsection{Case 3: diffusion equation}

If the convection term can be neglected, the diffusion convection Equation (70) can be simplified to diffusion equation

$$
\frac{\partial F}{\partial t}=\kappa_{z z} \frac{\partial^{2} F}{\partial z^{2}}
$$

Therefore, Equation (64) becomes

$$
\frac{d}{d t}\langle(\Delta z)\rangle=0
$$

Consequently, it is easy to see that the parallel diffusion coefficient can be shown as

$$
\kappa_{z z}=\frac{1}{2} \frac{d}{d t}\left\langle(\Delta z)^{2}\right\rangle
$$

\subsection{Case 4: the constant background magnetic field}

For the constant background magnetic field, the Fokker-Planck equation with time-dependent pitch-angle diffusion coefficient is

$$
\frac{\partial f}{\partial t}+v \mu \frac{\partial f}{\partial z}=\frac{\partial}{\partial \mu}\left[D_{\mu \mu}(\mu, t) \frac{\partial f}{\partial \mu}\right]
$$

By using the similar method in Section 2, from the latter equation we can obtain the variable coefficient differential equation of isotropic distribution function as

$$
\begin{aligned}
\frac{\partial F}{\partial t}= & \left(\kappa_{z z}(t) \frac{\partial^{2} F}{\partial z^{2}}+\kappa_{z z z}(t) \frac{\partial^{3} F}{\partial z^{3}}+\kappa_{z z z z}(t) \frac{\partial^{4} F}{\partial z^{4}}+\cdots\right)+\left(\kappa_{t z}(t) \frac{\partial^{2} F}{\partial t \partial z}+\kappa_{t t z}(t) \frac{\partial^{3} F}{\partial t^{2} \partial z}+\kappa_{t t t z}(t) \frac{\partial^{4} F}{\partial t^{3} \partial z}+\cdots\right) \\
& +\left(\kappa_{t z z}(t) \frac{\partial^{3} F}{\partial t \partial z^{2}}+\kappa_{t t z z}(t) \frac{\partial^{4} F}{\partial t^{2} \partial z^{2}}+\kappa_{t t t z z}(t) \frac{\partial^{5} F}{\partial t^{3} \partial z^{2}}+\cdots\right)+\cdots \cdots
\end{aligned}
$$

Comparing with Equation (25), we find that Equation (75) does not contain the convection term $\kappa_{z}(t) \partial F / \partial z$ which is caused by the adiabatic focusing effect. The first order moment of parallel displacement corresponding to Equation (75) is given as,

$$
\frac{d}{d t}\langle(\Delta z)\rangle=\kappa_{z}(t)=0
$$


Similarily, we can find the second order moment of the parallel displacement as

$$
\frac{d}{d t}\left\langle(\Delta z)^{2}\right\rangle=2 \kappa_{z z}(t)
$$

This equation can be rewritten as

$$
\kappa_{z z}(t)=\frac{1}{2} \frac{d}{d t}\left\langle(\Delta z)^{2}\right\rangle
$$

\section{SUMMARY AND CONCLUSION}

The parallel diffusion coefficient can be defined as one half of the temporal derivative of variance of the parallel displacement, DCDV (Equation (1)). It is shown that DCDV is only suitable to the transport equation without the convection term or without the cross terms. If the parallel diffusion coefficient $\kappa_{z z}$ is a constant, the formula $\sigma^{2}=2 \kappa_{z z} t$ holds, which means the transport regime is diffusion. The purpose of this work is to derive the formula of the parallel diffusion coefficient $\kappa_{z z}$ with the parallel displacement variance $\sigma^{2}$ for the more general transport equation of the isotropic distribution function. We start from the Fokker-Planck equation with time-dependent pitch-angle diffusion coefficient $D_{\mu \mu}(\mu, t)$, which is an extension of the Fokker-Planck equation with time-independent pitch-angle diffusion coefficient. From this equation, by employing the perturbation method which is frequently used in relevant research (see, e.g., Beeck \& Wibberenz 1986; Bieber \& Burger 1990; Schlickeiser \& Shalchi 2008; Schlickeiser \& Jenko 2010; Litvinenko \& Schlickeiser 2013; He \& Schlickeiser 2014; Wang et al. 2017b; Wang \& Qin 2018), the variable coefficient differential equation of the isotropic distribution function $F(z, t)$ with the time-dependent coefficients is derived. From the equation of $F(z, t)$ the modified formula describing the relation of the parallel diffusion coefficient $\kappa_{z z}$ with the parallel displacement variance $\sigma^{2}$ (MDCDV) is found. MDCDV indicates that the parallel diffusion is determined by not only the parallel displacement variance but also all the coefficients of the cross terms with the first order spatial derivative and convection term coefficient. Because all coefficients are time-dependent in MDCDV, the well-known formula $\sigma^{2} \propto t$ does not hold. Therefore, the transport of the energetic charged particles described by MDCDV is not in diffusion regime.

In addition, we study MDCDV in some special cases. For the isotropic distribution function equation with the constant coefficient, MDCDV is simplified to Equation (66). This formula demonstrates that the 
parallel diffusion coefficient is determined by the parallel displacement variance, the convection term coefficient, and the cross term coefficients with all the first order temporal and spatial derivatives. Since all the coefficients are constant, $\sigma^{2} \propto t$ can be found. Therefore, the constant coefficient differential equation of $F(z, t)$ can describe transport in diffusion regime. Furthermore, we explore the transport equation with uniform background magnetic field. For this case, the convection term does not occur in the equation of $F(z, t)$. Accordingly, MDCDV derived in this paper reduces to DCDV. For the diffusion convection equation and diffusion equation, the more simplified equations of $F(z, t), \mathrm{MDCDV}$ is also reduced to DCDV. The telegraph equation is another frequently used transport equation, which is derived by using iteration method from the isotropic distribution function equation. In this article, we also derive a new telegraph equation, and explore preliminarily the corresponding formula of $\kappa_{z z}$ with variance $\sigma^{2}$.

Instead of adibatic focusing, solar wind speed is another factor causing the convection term in the equation of the isotropic distribution function. So, when the phase space equation with solar wind speed is projected to real space, solar wind leads to a convection term in the real space equation. We will explore this topic in the future. As a subject of future work we will perform a detailed investigation into the new telegraph equation.

We are partly supported by grant NNSFC 41874206, NNSFC 41574172.

\section{REFERENCES}

Beeck, J., \& Wibberenz, G. 1986, ApJ, 311, 437

Bieber, J. W., \& Burger, R. A. 1990, ApJ, 348, 597

Earl, J.A. 1974, ApJ, 193, 231

Earl, J. A. 1976, ApJ, 205, 900

Earl, J. A. 1981, ApJ, 251, 739

Fedorov, Y. I., \& Shakhov, B. A. 2003, A\&A, 402, 805

Effenberger, F., \& Litvinenko, Y. E. 2014, ApJ, 783, 15

Hauff, T., \& Jenko, F. 2008, PhPl, 15, 112307

He, H.-Q., \& Schlickeiser, R. 2014, ApJ, 792, 85

Jokipii, J. R. 1966, ApJ, 146, 480
Kaghashvili, E. Kh., Zank, G. P., Lu, J. Y., \& Dröge, W. 2004, J. Plasma Phys., 7, 505

Kóta, J. 1994, ApJ, 427, 1035

Kóta, J. 2000, J. Geophys. Res., 105, 2403

Kunstmann, J. E. 1979, ApJ, 229, 812

Litvinenko, Y. E. 2012a, ApJ, 752, 16

Litvinenko, Y. E. 2012b, ApJ, 745, 62

Litvinenko, Y. E., Effenberger, F., \& Schlickeiser, R. 2015, ApJ, 806, 217 
Litvinenko, Y. E., \& Noble, P. L. 2016, Phys. Plasmas, 23, 062901

Litvinenko, Y. E., \& Schlickeiser, R. 2013, A\&A, 554, A59

Malkov, M. A., \& Sagdeev, R. Z. 2015, ApJ, 808, 157

Matthaeus, W. H., Gray, P. C., Pontius, D. H., Jr, \&

Bieber, J. W. 1995, PhRvL, 75, 2136

Matthaeus, W. H., Qin, G., Bieber, J. W., \& Zank, G. P. 2003, ApJ, 590, L53

Pauls, H. L., \& Burger, R. A. 1994, ApJ, 427, 927

Porrà, J. M., \& Masoliver, J. 1997, PhRvE, 55, 7771

Qin, G. 2007, ApJ, 656, 217

Qin, G., Matthaeus, W. H., \& Bieber, J. W. 2002a,

Geophys. Res. Lett., 29, 1048

Qin, G., Matthaeus, W. H., \& Bieber, J. W. 2002b,

ApJL, 578, L117

Qin, G., \& Zhang, L.-H. 2014, ApJ, 787, 12

Reimer, A., \& Shalchi, A. 2016, MNRAS, 456, 3803

Roelof, E. C. 1969, in Lectures in High Energy

Astrophysics, ed. H. Ögelmann \& J. R.

Wayland(NASA SP-199: Washington, DC: NASA), 111

Schlickeiser, R. 2002, Cosmic Ray Astrophysics

(Berlin: Springer)

Schlickeiser, R., Dohle, U., Tautz, R.C., \& Shalchi, A. 2007, ApJ, 661, 185

Schlickeiser, R., \& Jenko, F. 2010, J. Plasma Phys., 76, 317

Schlickeiser, R., \& Shalchi, A. 2008, ApJ, 686, 292

Schwadron, N. A., \& Gombosi, T. I. 1994, J. Geophys. Res., 99, 301
Shalchi, A. 2005, Phys. Plasmas, 12, 052905

Shalchi, A. 2009a, Nonlinear Cosmic Ray Diffusion Theories, Astrophysics and Space Science Library, Vol. 362 (Berlin: Springer)

Shalchi, A. 2009b, J. Phys. G: Nucl. Part. Phys., 36, 025202

Shalchi, A. 2010, ApJL, 720, L127

Shalchi, A. 2011, ApJ, 728, 113

Shalchi, A., Bieber, J. W., Matthaeus, W. H., \&

Schlickeiser, R. 2006, ApJ, 642, 230

Shalchi, A., \& Danos, R. J. 2013, ApJ, 765, 153

Shalchi, A., \& Kourakis, I. 2007, Phys. Plasmas, 14, 092903

Shalchi, A., \& Qin, G. 2010, Ap\&SS, 330, 279

Shalchi, A., \& Schlickeiser, R. 2005, ApJ, 626, L97

Shalchi, A., Škoda, T., Tautz, R. C., \& Schlickeiser, R. 2009a, A\&A, 507, 589

Shalchi, A., Škoda, T., Tautz, R. C., \& Schlickeiser, R. 2009b, PhRvD, 80, 023012

Wang, J.-F., Qin, G. 2018, ApJ, 868, 139

Wang, J.-F., Qin, G., Ma, Q.-M., Song, T., \& Yuan, S.-B. 2017a, Phys. Plasmas, 24, 082901

Wang, J.-F., Qin, G., Ma, Q.-M., Song, T., \& Yuan, S.-B. 2017b, ApJ, 845, 112

Wang, Y., \& Qin, G. 2016, ApJ, 820, 61

Zank, G. P., Lu, J. Y., Rice, W. K. M., \& Webb, G. M. 2000, J. Plasma Phys., 64, 507 